\title{
A Review Perennial Fruits Seed Production Potential Enhancement by Using Apomixes
}

\author{
${ }^{*}$ Yalew Teshome ${ }^{1} \quad$ Alemeneh Leweye ${ }^{2}$ \\ 1.Ethiopian Institute of Agricultural Research, Fogera National Rice Research and training Center, Woreta, \\ Ethiopia \\ 2.Ethiopian Fedral Democratic Republic Ministry of Agriculture, Addis Ababa, Ethiopia
}

\begin{abstract}
Globalization of agriculture is increasingly calling for improved efficiency and competitiveness of the existing production systems. Plants reproduce in different methods. Sexual reproduction of fruit trees is a rarely used method in horticulture. Mainly applied in research stations to conserve the richness of the gene pool and develop new varieties. On the production side, this method cannot satisfy the requirements for production quality and quantity. In other case plant can be asexually reproduced either by using part of two or more plants in a union or parts of the same plant which in the case of grafts age and in the rooting. All asexual propagation techniques belong one of the two categories. Seeds formed by apomixis have maternal genotype because their embryo is derived from that have not undergone of meiosis and fertilization that define sexual embryo development. Absence of meiotic process and paternal contribution to the embryo genotype do mean that apomixis offers a clonally propagating method of plants through seeds. As in most fruit tree species, walnut tree is a heterozygous plant, therefore the most certain way to get uniform plantations, with higher quality cultivars, is vegetative propagation. Vegetative propagation in walnut tree by grafting is still a difficult method that involves higher expenses. The most methods include Root Cuttings, Softwood cuttings, Hardwood cuttings, vegetative propagation by bud grafting or budding, Vegetative Propagation by grafting. Fruit formation by apomixy theoretical and practical significance; the embryo, being homozygous, is transmitting the similar characteristics of plant. (NICULINA, 2011). One of the most important prerequisites for genetic manipulation of plants in vitro is the ability to grow somatic cells in sterile plant growth medium and to regenerate viable plants from these cultures. Somatic embryogenesis, therefore, is a more efficient pathway for studies involving production of genetically transformed plants.(Kamle et al., 2011).
\end{abstract}

Keywords: embryo, grafting, propagation, rootstocks

DOI: $10.7176 / \mathrm{PPAR} / 11-9-02$

Publication date:May $31^{\text {st }} 2021$

\section{INTRODUCTION}

Globalization of agriculture is increasingly calling for improved efficiency and competitiveness of the existing production systems. The improvement of fruit trees through conventional breeding techniques has been limited due to inherent problems such as long life cycle with extended juvenile period, floral morphology, existing hybridization barrier, sterility, apomixes and long term inbreeding depression. Conventional propagation methods such as grafting, air layering, stooling etc. for improving many fruit trees already exist but extended juvenility has made these techniques time consuming and cumbersome. The attempt by Haberlandt to establish plant tissue culture systems provided support for a better understanding of the totipotency of plant cells. Plant tissue culture offers an effective solution of such problems of propagation of fruit crops. Improvement of fruit crops through several biotechnological approaches, highly efficient regeneration is a prerequisite.(Kamle, Bajpai, Chandra, Kalim, \& Kumar, 2011).

Apomixis is the natural ability of more than 400 plant species to reproduce asexually through seed .While sexual embryos result from the union of male and female gametes, which produce genetically varied offspring, apomictic embryos are formed directly from gametophytic or sporophytic ovarian tissue without paternal contribution. Apomixis may be obligate or facultative, resulting in either only apomictic produced embryos or seed, or a combination of apomictic and sexually-derived embryo and seed, In non-parthenocarpic Musa, unfertilized ovaries typically dehisce and die and do not produce mature fruit in a genotype-dependent developmental regime. (Length, 2011).

Most temperate tree fruit species are self- sterile and heterozygous and most scion cultivars do not come true-to-type if propagated using their own seeds. Consequently, when a tree raised from seed showed particularly desirable characteristics the only way of increasing it in number was by asexual (vegetative or clonal) propagation. Traditionally, cultivars of most tree fruit species propagate poorly on their own roots, irrespective of whether layering or cutting techniques are used. Although propagation research over the last 15 years has alleviated some of the problems associated with multiplying scions on their own roots poor cropping performance, suckering, and burrknotting continue to prove problems with self-rooted trees .(Webster et al. 1985). 
Early horticulturalists realized that the most obvious solution to the problem of propagating recalcitrant scions was to propagate them by budding or grafting onto more easily-propagated rootstocks. Initially, all rootstocks were raised from seed.

Fruits were collected from indigenous populations of native fruit species, the seeds extracted, germinated, and grown on for use as rootstocks. Suitable wild populations of apple (Malus domestic Borkh.), pear (Pyrus communis L.), plum (Prunus domestica L.), sweet cherry (Prunus avium L.), and sour cherry (Prunus cerasus L.) were abundant in many parts of Europe.(Webster \& Me, 2016).

Early propagators of fruit trees would have noted that graft compatibility, growth and cropping of scions raised on seedling rootstocks were often extremely variable. The rapid improvement in somatic embryogenesis methods allows the use of somatic embryos in plant micropropagation as synthetic seeds. (Burgos, 2005) .

OBJECTIVE

- To understand the meaning of apomixes

- To review the potential for seed production on perennials fruit trees

- To know different propagation techniques of perennial fruit trees

\section{Basic concepts of apomixes}

Apomixes, derived from two Greek word "APO" (away from) and "mixes" (act of mixing or mingling). It refers to the occurrence of an sexual reproductive process in the place of normal sexual processes involving reduction division and fertilization. In other words apomixes is a type of reproduction in which sexual organs of related structures take part but seeds are formed without union of gametes. Seeds formed in this way are vegetative in origin. When apomixes is the only method of reproduction in a plant species, it is known as obligate apomixes. On the other hand, if gametic and apomictic reproduction occurs in the same plant, it is known as facultative Apomixes The first discovery of this phenomenon is credited to Leuwenhock as early as 1719 in Citrus seeds.(Long \& An, 1954).

Apomixes is the formation of seeds by asexual processes. Apomixes comprises an ensemble of developmental processes that together alter the reproductive functions in the ovule of flowering plants, converting the sexual program me to an asexual one. The result of these alterations is that the apomictically derived embryo is not a product of male and female gamete fusion as occurs in sexual reproduction but is derived solely from cells in maternal ovule tissues.(Chaudhury, Scientific, \& Plant, 2001).

Sexual reproduction of plants like propagation from a cutting or leaves has never been considered as apomixis but replacement of a seed by a plantlet or replacement of a flower by bulbils is apomixis. Apomitically produced offspring are genetically identical to the parent plant. As apomictic plants are genetically identical from one generation to the next, each has the characters of true species. (Sarkar, 2011).

\section{Types of apomixes}

Mainly four types of apomixis phenomenon are suggested by Maheshwari (1954)

3.1. Recurrent Apomixis An embryo sac develops from the MMC or megaspore mother cell (archesporial cell) where meiosis is disturbed (sporogenesis failed) or from some adjoining cell (in that case MMC disintegrates). Consequently, the egg-cell is diploid. The embryo subsequently develops directly from the diploid egg-cell without fertilization. Somatic apospory, diploid parthenogenesis and diploid apogamy are recurrent apomixis. However, diploid parthenogenesis / apogamy occur only in aposporic (somatic) embryo-sacs. Therefore, it is the somatic or diploid aposory that constitutes the recurrent apomixis. Such apomixis occurs in some species of Crepis, Taraxacum, Paa (blue grass), and Allium (onion) without the stimulus of pollination. Malus (apple), and Rudbeckia where pollination appears to be necessary, either to stimulate embryo development or to produce a viable endosperm.

3.2. Non -recurrent Apomixis An embryo arises directly from normal egg-cell (n) without fertilization. Since an egg- cell is haploid, the resulting embryo will also be haploid. Haploid parthenogenesis and haploid apogamy, and androgamy fall in this category. Such types of apomixis are of rare occurrence.

3.3. Adventives Embryony Embryos arise from a cell or a group of cells either in the nucleus or in the integuments, e.g. in oranges and roses. Since it takes place outside the embryo sac, it is not grouped with recurrent apomixis, though this is regenerated with the accuracy. In addition to such embryos, regular embryo within the embryo sac may also develop simultaneously, thus giving rise to poly embryony condition, as in Citrus, Opuntia.

3.4. Vegetative apomixis In some cases like Poa bulbosa and some Allium, Agave and grass species, vegetative buds or bulbils, instead of flowers are produced in the inflorescence. They can also be reproduced without 
difficulty. However, Russian workers do not group this type of vegetative reproduction with apomixis. Now, different apomictic phenomena in each of the recurrent and non-recurrent apomicts are considered in relation to the development of the embryo sac or embryo.(Long \& An, 1954).

\section{Mechanisms of Apomixis}

In contrast to sexual seed formation, apomixis can occur by various mechanisms that share three common develop-mental components: (i) a bypass of meiosis during embryo sac formation (apomeiosis), (ii) development of an embryo independent of fertilization (a process known as parthe-nogenesis), and (iii) formation of viable endosperm either via fertilization-independent means or following fertilization with a sperm cell.

Derivation of the egg from a diploid maternal cell without meiotic reduction, and its subsequent fertilization-independent development into an embryo, means that the progeny derived from apomictic development are clonal and therefore genetically identical to the maternal parent.

Apomixis mechanisms are historically subdivided into two categories and classified as either gametophytic or sporophytic, based on whether the embryo develops via a gametophyte (embryo sac) or directly from diploid somatic (sporophytic) cells within the ovule. During sporophytic apomixis, development of an embryo sac following the typical angiosperm sexual pathway still occurs. However, during mitosis of the functional megaspore, diploid somatic ovule cells surrounding the embryo sac differentiate and have an embryogenic cell fate. These embryo initial mother cell position). This cell may enter meiosis and abort the process or it may immediately begin mitosis. (Hand \&Koltunow,2014).

\section{Advantages of apomixis}

In plant breeding the two sexual processes, self-and cross fertilization, followed by segregation, tend to alter the genetic composition of plants reproduced through apomoxis. Inbreeding and uncontrolled out breeding also tend to break heterozygote superiority in such plants. On the contrary, apmicts tend to conserve the genetic structure of their carriers. They are also capable of maintaining heterozygote advantages generation after generation. Therefore, such a mechanism might offer a great advantage in plant breeding where genetic uniformity maintained over generation for both homozygosity (in varieties of selfers), and heterozygosity (in hybrids of both seIfers and outbreeders) is the choicest goal. Thus, in short the benefits of apomixis, insofar as their utility in plant breeding is concerned, are:

1. Rapid multiplication of genetically uniform individuals can be achieved without risk of segregation.

2. Heterosis or hybrid vigor can permanently be fixed in crop plants, thus no problem for recurring seed production of $\mathrm{F} 1$ hybrids.

3. Efficient exploitation of maternal effect, if present, is possible from generation to generation.

4. Homozygous inbred lines, as in corn, can be rapidly developed as they produce sectors of diploid tissues and occasional fertile gametes and seeds. (Long \& An, 1954).

\section{Importance of Apomixis to Agriculture}

Apomixis is of great importance to agriculture primarily because of its potential to facilitate multiplication of F1 hybrid seeds. Hybrid seeds are preferred for use in modern agriculture mainly because they provide a yield advantage.

Due to these and other potential benefits of apomixis in agriculture, apomixis is likely to be an important breeding tool for the future. Little is known about the genes controlling apomixis. Research is currently being pursued to isolate these genes and to understand how the process of apomixis can be regulated to result in high yields of clonal seed. The value of apomixis will only be realized if it can be efficiently controlled under conditions of commercial production.(Chaudhury et al., 2001).

Many agronomic advantages of apomixis can be envisioned: the rapid generation and multiplication of superior forms through seed from novel, currently underused germplasms; the reduction in cost and time of breeding; the avoidance of complications associated with sexual reproduction, such as pollinators and crosscompatibility; and the avoidance of viral transfer in plants that are typically propagated vegetatively, such as potatoes .(Bicknell \& Koltunow, 2004).

\section{Impact on seed industry}

Experts on apomixes have repeatedly mentioned the simplification of breeding procedures for grain crops as the major potential advantage of apomixis. It seems, however, that apomixes will first need to be transferred to a major crop before breeders begin to reexamine the field and how apomixis may be applied to their particular species. The advent of individual hybrid plant selection, in a world of sexual crops, where improvement has heretofore been on family based strategies, will enable scientists to create totally new breeding schemes, much more related to those used in clonal crops.

Some disadvantages could be that the farmers could save their own seed instead of purchasing new seed 
each year and another concern is the control of rights to germplasm. Documentation method would need to be refined and precise because of proliferation of cultivars in the markers, some with only small genetic differences.(Pablo, 2002) .

\section{Potential seed production in perennial fruit tree 8.1. History of rootstock propagation}

Perennial fruits can be propagated in vegetative or generative ways. Fruit and nut trees are usually propagated by vegetative means using grafting methods. For this, there are two types of grafting methods: budding and grafting. Both of them are used for the same purpose -to create a new plant through the union of a suitable rootstock and an aerial part of another plant of the desired variety, called a scion. Other fruits are propagated by their own cuttings, such as stem, root and runner. (Crop \& Series, 2007).

Sexual reproduction of fruit trees is a rarely used method in horticulture. Mainly applied in research stations to conserve the richness of the gene pool and develop new varieties. On the production side, this method cannot satisfy the requirements for production quality and quantity. Plant can be asexually reproduced either by using part of two or more plants in a union or parts of the same plant. In the first case we call it graft age and in the second case we call it rooting. All asexual propagation techniques belong one of the two categories.

Rootstocks have been used for propagating fruit trees for more than 2000 years; ancient manuscripts record their use as far back as the Hellenistic period in Greek history (300-30 BC). For approximately the first two millenia since their first use the sole reason for rootstocks was as a means of propagating the scion. Most temperate tree fruit species are self- sterile and heterozygous and most scion cultivars do not come true-to-type if propagated using their own seeds. Consequently, when a tree raised from seed showed particularly desirable characteristics the only way of increasing it in number was by asexual (vegetative or clonal) propagation. Traditionally, cultivars of most tree fruit species propagate poorly on their own roots, irrespective of whether layering or cutting techniques are used. Although propagation research over the last 15 years has alleviated some of the problems associated with multiplying scions on their own roots (Jones et al. 1985), poor cropping performance, suckering, and burrknotting continue to prove problems with self-rooted trees (Webster et al. 1985).

Early horticulturalists realized that the most obvious solution to the problem of propagating recalcitrant scions was to propagate them by budding or grafting onto more easily-propagated rootstocks. Initially, all rootstocks were raised from seed.

Fruits were collected from indigenous populations of native fruit species, the seeds extracted, germinated, and grown on for use as rootstocks. Suitable wild populations of apple (Malus domestica Borkh.), pear (Pyrus communis L.), plum (Prunus domestica L.), sweet cherry (Prunus avium L.), and sour cherry (Prunus cerasus L.) were abundant in many parts of Europe.

Early propagators of fruit trees would have noted that graft compatibility, growth and cropping of scions raised on seedling rootstocks were often extremely variable. Trees on a few of the seedling rootstocks would have been smaller in stature or shown other differences in habit, flowering and/or fruiting. At an early stage the potential benefits of some of these rootstock effects on scion growth would have been recognized and the first clonal rootstocks selected and propagated. Quite when this began is uncertain, although records suggest that clonal rootstocks were in use as early as the 17th Century. To be of any value to the early fruit tree propagator the selected clonal rootstocks must themselves have been easy to propagate. Many would have been dug up as suckers from around fruiting orchard trees; later, techniques of layering or stooling were employed for their propagation.

For many years only those clones which could be propagated easily from layering/stooling techniques were selected as clonal rootstocks. It is only relatively recently that attributes other than ease of propagation have become important considerations in clonal rootstock selection.

\subsubsection{Methods of rootstock propagation}

The principal methods employed for rootstock propagation are shown in Table 1. Throughout the World most rootstocks are raised from seed, although an increasing number of clonal rootstocks are now used for the propagation of apple, pear, and sweet cherry cultivars. Most clonal apple and sweet cherry rootstocks are propagated by division techniques (stooling or layering); relatively small numbers are raised by cutting techniques, including micro-propagation and root cuttings. Where a rootstock clone has particularly desirable qualities but proves difficult to root, it may occasionally be used as an inter stock, grafted between the scion and some more easily-propagated rootstock clone. 


\begin{tabular}{|l|l|l|}
\hline \multicolumn{2}{|l|}{ Propagation their owen } \\
\hline Natural & Asexual seedlings & apomixis - mangosteen polyembryony - mango, citrus \\
\hline \multirow{2}{*}{ Man-made } & Rooting after separation (cuttings) & Root cuttings - breadfruit, plum Stem cuttings - grape \\
\cline { 2 - 3 } & Rooting on mother plant & Layering - guave, blackberry Air layering - longan, lime \\
\hline Propagation on rootstock & \multicolumn{2}{|l|}{} \\
\hline Budding & $\begin{array}{l}\text { T-budding - citrus, apple Chip-budding - citrus Patch- } \\
\text { budding - avocado, rubber }\end{array}$ \\
\hline Grafting & On rootstock in the nursery & $\begin{array}{l}\text { Tip grafting - young, tender stock Side grafting - mature, } \\
\text { woody stock }\end{array}$ \\
\hline
\end{tabular}

Table 1: rootstock propagation method

\section{A. Propagation by seed}

The great majority of rootstocks for many temperate and subtropical fruit and nut species, including peaches, nectarines (Prunus persica L.), apricots (P. armeniaca L.), asian pears (Pyrus pyrifolia (Nakai)), and citrus species are raised by seed. Propagation by seed has significant advantages for the nurseryman; in particular, it is both simpler and cheaper to achieve than propagation by vegetative methods. Whether trees propagated on seedling rootstocks have any advantages to the fruit grower, however, is much less clear and in many instances trees on seedling rootstocks are greatly inferior to those on clonal rootstocks.

In species such as apple, where viruses are thought not to be transmitted through seed and where nurseries in some parts of the world find it difficult to maintain the health status of virus-free clonal rootstocks, seedling rootstocks may have clear benefits. Seedling propagation also offers the potential for avoiding transmission of root-borne diseases such as crown gall (Agrobacterium tumefaciens). Rootstock liners from infected stool or layer beds frequently transfer this troublesome disease to the new scion tree and the new site.

Seedling stocks raised on soils free of crown gall avoid this problem. Unless clonal rootstocks have been selected which confer other benefits to the scion, such as reducing vigor of growth, inducing early and abundant cropping, or conferring resistance to soil-borne pests and/or diseases, then there are less benefits gained by changing from seedling to clonal rootstocks. Nevertheless, seedlings of many heterozygous, out-crossing fruit species are extremely variable in performance when used as rootstocks and one clear benefit of changing to a clonal rootstock is the improved uniformity of growth and cropping in the scion. It is unfortunate that the change from seedling to clonal rootstocks is invariably resisted by nurserymen for both economic and logistic reasons.

The uniformity of performance of seedling rootstocks may, however, be improved to some extent by: (1) using seed of a single clonal variety (e.g., 'Red Delicious' apple or 'Bartlett' pear) or seed from a self-fertile cultivar grown in a monoculture; (2) using seed collected from virus-free mother orchards planted in isolation .One problem was that the apple apomicts were of the facultative type, producing zygotic as well as apomictic seeds in their fruits. Culling these out at the nursery stage proved difficult. Other reasons for their poor acceptance were their strong vigor when used as rootstocks, incompatibility with some scions and sensitivity to virus infection.

In comparison with vegetative propagation relatively little research is now conducted into the techniques of seedling rootstock propagation for temperate fruits. The techniques of after-ripening and dormancy-breaking, essential with seed of many Rosaceae, are now well elucidated. Aids to dormancy-breaking, such as scarification, and stratification are widely adopted by commercial nurserymen.(Webster \& Me, 2016).

Treatments with hormones, which may also aid dormancy breaking, are less frequently adopted, however. Treatment with gibberellic acid (GA3) and benzyl adenine (BA), (both at $20 \mathrm{mg} /$ litre), of peach seeds which had previously been stratified at 10 and $15^{\circ} \mathrm{C}$ enhanced germination, whereas treatment with thiourea was ineffective in Thai trials (Siyapananont 1990). Similar results were recorded by Shatat \& Sawwan (1985) who demonstrated that germination of Prunus mahaleb seed was improved significantly by treatment with Promalin (a mixture of GA4+7 and BA) at $3000 \mathrm{mg} /$ litre. Research in Poland on germination of plum rootstock seeds (Grzyb \& Czynczyk 1990) showed that soaking seed in a solution of $500 \mathrm{ppm}$ ethephon just before sowing improved germination. This work also demonstrated that autumn sowing of partially stratified seeds produced better results than spring sowing of fully stratified seeds.

Many innovative changes in seedling rootstock propagation now focus on the development of improved machinery for undertaking the various operations associated with land preparation, seed sowing, seedling management, and lifting operations.(Webster \& Me, 2016).

\section{B. Stooling or layering}

Stooling or layering, the division techniques used in tree rootstock propagation, were ably described many years ago (Knight et al. 1927) and little of significance has since changed in how these techniques are executed. Rooting is usually stimulated by excluding light from the targeted section of stem, either by blanching or etiolating; this is achieved using soil, another medium (sawdust/peat), or covering with an opaque material such 
as plastic. Also necessary are adequate temperatures coupled with sufficient moisture and oxygen at the edaphic zone immediately peripheral to the targeted rooting zone.

Stool and layer beds are also subject to attack from numerous soil-borne pests and diseases. Many nematode species severely limit production, as do attacks by bacterial (e.g., Agrobacterium spp.) and fungal (e.g., Thielaviopsis, Phytophthora spp.) diseases. The most productive rootstocks tended to produce the highest number of shoots/m and the amount of root formation was greatest on shoots of the most productive rootstocks. The main purpose of layering is to provide rooting for the stem of the mother plant. The new growing plant will keep the union with the mother plant until it is able to survive on its own. When this happens, the new plant will be cut off from the mother plant. There are different techniques of layering this includes air layering, banking up, simple layering, radial layering.

\section{Air Layering}

This method is used on the tip of the branch, when stems are usually younger then one year old. A strip of bark is cut approximately $2 \mathrm{~cm}$ wide on the stem about $20 \mathrm{~cm}$ from the tip (just below a leaf stalk, or join). Once cut, a rooting hormone is applied and rooting material is placed under the strip. Finally, the cut is covered with a thin plastic bag, which is opened at both ends. Rooting material must be placed in the bag before it is sealed. This process must be completed during rainy conditions, when the air humidity is highest. Litchi, guava, macadamia and mango are propagated with this method.

Problems with stooling and layering are usually attributable to the above requirements not being properly met. Poor natural soil conditions or the use of inappropriate media for earthing-up stool or layer beds are a common reason for poor stool or earthing-up stool/layer beds in many parts of the world and care should be taken that the wood species used contain no chemicals, natural or introduced, likely to be inhibitory to stool shoot rooting. Some nurserymen in Britain have incorporated a layer of moist peat close to the base of the shoots to encourage stool shoot rooting. Delays in earthing-up and insufficient moisture are other common problems which frequently lead to poor rooting.

\section{Banking Up}

This is the most common method to propagate pear, quince and apple rootstock (M type clones). This technique needs some preparative work. For about 2-3 years, we cut back the mother plant up to the surface level (or close to it), which will then result in a thicker root neck. This thick root neck will grow custard of stems. We then have to bank up the plant to $10-15 \mathrm{~cm}$ high (when the stems have an average $20-25 \mathrm{~cm}$ length). We can subsequently harvest the rooted stems at the end of the growing season.

\section{E. Simple Layering}

This technique is commonly used for hazel-nut propagation. During the dormant season, stems are bent down into a $20-25 \mathrm{~cm}$ deep trench and covered with soil. The top parts of the stems, which usually have 2-3 buds on them, remain above the surface.

\section{F. Propagation by cuttings}

Most new research on rootstock propagation in recent years has focused on improving the success achieved with conventional cutting techniques and developing the allied technique of micropropagation. Usually, cutting techniques have been developed to aid the propagation of recalcitrant rootstock clones or to circumvent disease, soil or site problems experienced with the more conventional division techniques.

In Britain, much research work has focused on developing successful hardwood cutting techniques for apple, plum, and quince (Cydonia oblonga Mill.) rootstock clones. Elsewhere, methods softwood cutting propagation, usually based on intermittent misting techniques, has been developed for many Prunes rootstock clones. Recently, fogging systems have been shown to give better results than misting techniques with softwood cuttings of some species.(Crop \& Series, 2007).

For successful propagation using any type of cutting there are three principal considerations. First, the propagule must be healthy and in the appropriate physiological condition; this is achieved by good stock plant management before cutting excision. Second, the cutting may need physical or chemical treatment to aid its rooting and, finally, the cutting must be placed in an environment conducive to survival, root induction, and/or root development.

\section{Vegetative Propagation by Grafting}

Two types of rootstock can be used for grafting: the cultivar and the seedling rootstock. The cultivar rootstock is produced by vegetative methods, generally by layering and cuttings. Seedling rootstocks grow from seed. One of the best examples for cultivar rootstock is the apple and for the seedling rootstock, the mango .During the selection of the scion wood we have to consider some important aspects:

\subsection{Whip Grafting}

The whip graft is useful for plants that unite easily. This method is useful for apples, mangos and pears. It can be used to graft root, stem or top graft. The diameter of the scion and rootstock should be the same, from the size of 
a pencil to $10-15 \mathrm{~mm}$

\subsection{Cleft Grafting}

This method has been practiced throughout the history of horticulture and is one of the oldest fruit propagation techniques. It is suited for apple and pears, but, in tropical areas, in can also be used for propagation of mango and avocado trees. Citrus and guava trees also use this method. In the case of top and side work, the scaffold limb is usually wider than the scion. In the case of tree propagation, both parts, the rootstock and scion, should be the same size.

\subsection{Bark Grafting}

Bark grafting is used when the stock is too large for whip grafting. It is one of the most difficult grafting techniques. Perfect application of this method requires much practice and experience. The use of this technique is common for pear, apple and different nuts grafting.

\subsection{Approach Grafting}

Some trees are very difficult to graft such as mango and macadamia. In these cases we can use the approach grafting method. The main difference between these techniques and other methods are that the scion is attached to its root system during the grafting process. Take two plants. One will be the rootstock and the other the scion. Make the same cut on both stems at the same height. Hold the two stems together and tie them with tape. When the grafts have joined, remove the top of the rootstock plant with a cut above the joining point and remove the bottom of the scion plant with a cut below the joining point.

\subsection{Micropropagation}

Micropropagation has become fashionable in recent years and many commercial micropropagation laboratories have targeted rootstocks as suitable subjects. In the last 10 years new and improved micropropagation techniques have been developed for many rootstocks including the apple clones.

In some instances, where micropropagation is the only method by which the clone may be propagated, its use is entirely justified. The technique also has value in rapidly building up new rootstock types or in facilitating the movement of healthy materials over national borders whilst satisfying plant importation and health regulations.

Micropropagation has proved particularly valuable in aiding the propagation of recalcitrant rootstocks, such as the apple clones.(Webster \& Me, 2016).

\subsection{Somatic embryogenesis}

Somatic embryogenesis (SE) is a process in which bipolar structures resembling a zygotic embryo, develops from a non zygotic cell without vascular connections with the original tissue. Somatic embryo are used for studying regulation of embryo development but, embryogenesis is a multistep regeneration process starting with the formation of proembryogenic masses followed by somatic embryo formation maturation, desiccation and plant regeneration. It is an important system where multiplication can be done at enormous rates. Somatic embryogenesis involves the production of embryo-like structures from somatic cells without gametes fusion. During their development, somatic embryos pass through stages similar to those observed in zygotic embryogenesis. It involves control of 3 consecutive steps: (i) induction of embryogenic lines from explant (ii) maintenance and multiplication of embryogenic lines; (iii) maturation of somatic embryos and conversion into viable plantlets. In somatic embryo, somatic cells develop are induced to form complete embryo similar to that of zygotic embryo. Both embryos basically undergo the same stages of development namely globular, heart shaped, torpedo, cotyledonary and mature embryos. They arise naturally in some species in a process known as direct somatic embryogenesis. In contrast, somatic embryos develop from in vitro cultured cells in the process called indirect somatic embryogenesis. Somatic embryos can differentiate either directly from the explant without an intervening callus phase or indirectly after a callus phase The multiplication step has been comparatively less investigated although it directly contributes to the final plant yield and influences the ability of the resulting embryos to germinate and develop into growing plantlets.

\section{a. Significance of somatic embryogenesis}

In recent years, development of plant cell, tissue culture technique has a considerable potential for the improvement of several fruit trees. Somatic embryogenesis is a developmental process of somatic cells, which resembles morphologically zygotic embryogenesis.

It is an important pathway for regeneration of plants from cell culture system and a method commonly used in large scale production of plants and synthetic seeds. In most of the important fruit crops, tissue culture is well established for plant regeneration via somatic embryogenesis. Many workers have emphasized somatic embryogenesis as a preferred method for genetic improvement and multiplication of valuable germplasm of a 
number of woody perennials. Since somatic embryo culture soften originate from a single cell, it is an ideal system for induction of mutations as it helps in preventing chimeras.

The process of somatic embryogenesis is not only important for the production of plants and secondary products, but also for the transgenic plants and somatic cell genetics. It plays an important role in clonal propagation. When integrated with conventional breeding programs and molecular and cell biological techniques, somatic embryogenesis provides a valuable tool to enhance the pace of genetic improvement of commercial crop species . However, somatic embryogenesis has other practical applications in crop improvement (cell selection, genetic transformation, somatic hybrid and polyploid plant production), germplasm preservation, virus elimination, in vitro metabolite production, and in vitro mycorrhizal initiation.

\section{b. In vitro selection}

Tissue-cultured cell lines can be selected in vitro for resistance to various stresses. Tissue culture techniques have been widely used for breeding purposes, especially in selection for stress tolerance. Selection is done by placing a stress causing agent in tissue cultures containing dividing cells. An efficient method for obtaining plants with desired characteristics is to add a selective agent that will kill the majority of the cells (except the resistant ones) to a tissue culture. This procedure is called in vitro selection. Since the in vitro unit of selection can be a single cell the selection pressure can be uniformly and reproducibly applied. Also, in vitro selection is potentially more efficient than whole plant selection. It is a source of genetic variability that gives rise through genetic modifications during the process of in vitro culture to a phenomenon called somaclonal variation. In vitro selection offers an immense potential for the quick and comprehensive generation of useful somaclones or mutants for resistance against various biotic and a biotic factors. This method has been particularly effective for selecting herbicide tolerant and disease-resistant plants.

\section{c. Genetic transformation}

Genetic transformation offers the opportunity for genetic manipulation of plants at cellular level and provides the means for modifying single horticultural traits without. The main target of gene transfer techniques is to produce improved varieties through the incorporation of important genes into existing cultivars. However, the plant transformation techniques do not transform all cells, and plants regenerated from transformed tissues via organogenesis are often chimeras.

Somatic embryos arise from single cells and for this reason regeneration via somatic embryogenesis reduces the formation of chimeras. Nevertheless, somatic embryogenesis seems Fruit trees are considered to be recalcitrant material for genetic transformation studies and the main impediment for genetic transformation is the regeneration of transformed plantlets. Choice of explants having competence for transformation and regeneration is a crucial factor. Hence, efficient tissue culture techniques become the base for genetic transformation studies. The successful regeneration of genetically transformed plants has been achieved in several tropical fruit crop species.

Applications of genetic transformation technology hold greater potential for fruit crops or perennials than even the herbaceous plants because the gains in genetic improvement can be realized over relatively short periods compared to routinely used techniques of hybridization, selection and mutagenesis which require decades.

The present area of research depends on at least three factors that must come together for each species or genotype to be improved: (1) availability of specific genes and promoters for transfer and expression; (2) development of reliable techniques for transfer of the genes to target cells and the selection of transformed cells; and (3) regeneration of transformed cells into whole plants which can be mass-propagated, preferably by asexual means. A variety of potentially useful genes is being characterized and cloned for transfer to agriculturally important plants, many of which also will be useful for the improvement of fruit crops.

\section{Potential applications of somatic embryogenesis}

1) Mass scale production: Somatic embryos have a great advantage over conventional breeding of propagation. Tremendous amount of embryos can be produced from single explant.

2) Rapid Multiplication: Rapid multiplication through production of somatic embryogenesis in cell cultures, and use of bioreactors for scale-up technology.

3) Shortening of breeding cycle: The process shortens the breeding cycle of tree species and thus increases the germination of hybrid embryos.

4) Transgenics: Development of somatic embryogenesis pathway could lead to exploitation of transgenic against various biotic and abiotic stresses.

5) Molecular and biochemical: Provides important source for the analysis of molecular and biochemical events that occur during induction and maturation of embryos.(Kamle 2011).

\section{CONCULSION}

Seeds formed by apomixis have maternal genotype because their embryo is derived from cells that have not 
undergone the events of meiosis and fertilization that define sexual embryo development. Absence of meiotic process and paternal contribution to the embryo genotype do mean that apomixis offers a clonally propagating method of plants through seeds. As in most fruit tree species, walnut tree is a heterozygous plant, therefore the most certain way to get uniform plantations, with higher quality cultivars, is vegetative propagation. Vegetative propagation in walnut tree by grafting is still a difficult method that involves higher expenses. Research conducted in walnut tree on fruit formation has shown that some cultivars can form fruit even without fecundation, by parthenocarpic or apomictic way. Opportunity of fruit formation without pollination in walnut, under normal conditions, has raised special interest for researchers in flowering biology and seed formation. Fruit formation by apomixy has theoretical and practical significance; the embryo, being homozygous, is transmitting the similar characteristics of mother plant. ( niculina,2011).

Clonal propagation is the key to intensification and higher yield: fewer unproductive years, more trees per ha, higher maximum yield per ha, much higher mean yield over the orchard's lifespan, more efficient management and lower cost of production.(Verheij, 2006).

Somatic embryogenesis is a promising method for the establishment of protocols for rapid multiplication of new and elite genotypes, synthetic seed production, in vitro selection approaches for various biotic and abiotic stresses and for studies of genetic manipulation. Gene transfer into embryogenic plant cells is already challenging conventional plant breeding, and has become an indispensable tool for crop improvement. One of the most important prerequisites for genetic manipulation of plants in vitro is the ability to grow somatic cells in sterile plant growth medium and to regenerate viable plants from these cultures. Somatic embryogenesis, therefore, is a more efficient pathway for studies involving production of genetically transformed plants.

\section{References}

Kamle, M., Bajpai, A., Chandra, R., Kalim, S., \& Kumar, R. Somatic embryogenesis for crop improvement, 2(June 2011), 54-59.

Length, F. Genetic evidence of developmental components of parthenocarpy in Apomictic Musa species, 3(August2011), 138-145.

Webster, A. D., \& Me, K. Temperate fruit tree rootstock propagation, 0671(March2016). http://doi.org/10.1080/01140671.1995.9513912

Burgos, C. P. and L. Advances and Future Perspectives in Fruit Tree Transformation, (May2005), 9-11

Long, A., \& An, R. A. Apomixis - classification and significance inplant breeding(1954),03

Chaudhury, A., Scientific, C., \& Plant, C. Apomixis(2001), 1-3.

Brazilian, C., Maria, D., Dusi, A., \& Agricultural, B. Apomixis: Occurrence, Applications Improvements Apomixis : Occurrence, Applications and Improvements, (March 2016).

Hand, M. L., \& Koltunow, A. M. G. The Genetic Control of Apomixis : Asexual Formation, 197(June2014), 441-450. http://doi.org/10.1534/genetic 114.163105

Cosmulescu, S., Botu, M., \& Achim, G. Determination of Apomictic Fruit Set Ratio

Several Romanian Walnut ( Juglans regia L .) Cultivars, 40(1)(,2012) 229-233. Sarkar, J. Vigour maintaining seeds for farmers,(2011) 101(1)

Chaudhury, A., Scientific, C., \& Plant, C. (2001). Apomixis, 1-3.

Bicknell, R. A. \& Koltunow, A. M. Understanding Apomixis: Recent Advances RemainingConundrums(2004 )16, 228-246. http://doi.org/10.1105/tpc.017921.Apomixis

Pablo, D. Breeding in Apomictic Species(2002)

Crop, P., \& Series, S. vegetative propagation techniques perren crop support series(2007)

kr chapmam, M. pinto. Pereninal Tropical Fruit Development(1992) .

Niculinia , C. S. Research on Fruit Formation by Apomixis in local Walnut tree cultivalters Project manager :(2011), 1063

Verheij, E. Fruit growing in the tropics.(2006) 